\title{
Rotational excitation of SiO by collisions with helium
}

\author{
F. Dayou and C. Balança
}

\begin{abstract}
Laboratoire d'Étude du Rayonnement et de la Matière en Astrophysique, UMR 8112 du CNRS, Observatoire de Paris-Meudon, 5 place Jules Janssen, 92195 Meudon Cedex, France e-mail: [fabrice.dayou; christian.balanca]@obspm.fr
\end{abstract}

Received 29 May 2006 / Accepted 4 July 2006

ABSTRACT

\begin{abstract}
Context. Within shocked regions of the interstellar medium and circumstellar environment of AGB stars the proper modelling of $\mathrm{SiO}$ line emission through non-LTE radiative transfer calculations requires accurate values of collisional rate coefficients.

Aims. The present study focuses on the transitions among the rotational levels of the $\mathrm{SiO}$ molecule in its ground vibrational state induced by collision with $\mathrm{He}$. The $\mathrm{H}_{2}$ molecule being the main colliding partner for the astrophysical regions of interest, the collisional process between $\mathrm{SiO}$ and para- $\mathrm{H}_{2}(j=0)$ is also investigated in an approximated way.

Methods. A new 2D SiO-He potential energy surface is computed by means of highly correlated ab initio calculations. Collisional rate coefficients corresponding to the pure rotational (de)excitation of $\mathrm{SiO}$ by collision with $\mathrm{He}$ are obtained from close-coupling quantum scattering calculations of inelastic cross sections. The $\mathrm{SiO}-\mathrm{He}$ potential energy surface is also employed to compute rate coefficients for the rotational (de)excitation of $\mathrm{SiO}$ by collision with para- $\mathrm{H}_{2}(j=0)$.

Results. Rate coefficients for rotational levels up to $j=26$ and kinetic temperatures in the range 10-300 K are obtained for the $\mathrm{SiO}-\mathrm{He}$ colliding system. The large asymmetry of the $\mathrm{SiO}-\mathrm{He}$ potential energy surface induces a propensity rule that favours odd $\Delta j$ transitions over even $\Delta j$. The estimated values of the $\mathrm{SiO}$-para- $\mathrm{H}_{2}(j=0)$ rate coefficients are compared with those of Turner et al. (1992) for the twenty first rotational levels. As a result of significant differences between the SiO-He interaction potentials employed in the two studies, the rate coefficients are found to differ by a factor $2.5-5$ for the main rotational transitions, whatever the temperature range.
\end{abstract}

Key words. ISM: molecules - molecular processes - molecular data

\section{Introduction}

The silicon monoxide $(\mathrm{SiO})$ molecule was detected for the first time in the interstellar medium by Wilson et al. (1971) through the $J=3 \rightarrow 2$ radio line emission from the galactic source Sagittarius B2, shortly after the first detection of the interstellar CO molecule (Wilson et al. 1970). Since then, $\mathrm{SiO}$ has been detected in a variety of astrophysical objects, and the modelling of observed molecular spectra showed that the $\mathrm{SiO}$ molecule, in contrast to $\mathrm{CO}$, is mostly present in regions associated with warm, dense, and shocked gas. In quiescent cold clouds the $\mathrm{SiO}$ abundance is less than $10^{-12}$ relative to $\mathrm{H}_{2}$ (Ziurys et al. 1989), but it can increase by several orders of magnitude in high velocity molecular outflows from young stellar objects (Martin-Pintado et al. 1992; Acord et al. 1997; Codella et al. 1999; Nisini et al. 2002). To date, the most likely explanation of such an enhancement of the $\mathrm{SiO}$ abundance comes from the sputtering of dust grains in shocked regions and the subsequent release of Si-bearing material into the gas phase (Flower \& Pineau des Forets 1995; Schilke et al. 1997; Le Picard et al. 2001; Dayou \& Spielfiedel 2003; Jiménez-Serra et al. 2005). Rotational line emissions of $\mathrm{SiO}$ are thus currently used as tracers of shocked gas in molecular outflows surrounding low- and high-mass star-forming regions (Martin-Pintado et al. 1992; Acord et al. 1997; Gueth et al. 1998; Codella et al. 1999; Nisini et al. 2002), as well as shocked regions of galactic nuclei (Martin-Pintado et al. 1997; Usero et al. 2006). The fact that Si-bearing species probably are major components of dust grains for an oxygen-rich composition of the gas
(Jeong et al. 1999) also makes $\mathrm{SiO}$ very useful for gaining information about the formation and evolution of dust grains. $\mathrm{SiO}$ radio line emissions detected towards outer atmospheres and circumstellar envelopes (CSE's) of Asymptotic Giant Branch (AGB) stars, especially M-type stars $(\mathrm{C} / \mathrm{O}<1)$, are thus employed as a probe of circumstellar dust formation and dynamics (Bieging et al. 2000; González Delgado et al. 2003; Schöier et al. 2004), shedding light on the mechanisms responsible for the mass-loss of AGB stars.

Within shocked regions of the interstellar medium and circumstellar environment of AGB stars, the proper modelling of $\mathrm{SiO}$ line emission through non-LTE radiative transfer calculations requires accurate values of collisional rate coefficients with $\mathrm{H}_{2}$, the most abundant molecule, and to a lesser extent with $\mathrm{H}$ and $\mathrm{He}$. In most cases the observed $\mathrm{SiO}$ line emissions involve rotational transitions within the ground vibrational state of $\mathrm{SiO}$, although vibrationally excited states of $\mathrm{SiO}$ may also have to be considered in the case of circumstellar $\mathrm{SiO}$ maser line emissions from evolved stars (Pardo et al. 1998; Herpin et al. 2006; Chen et al. 2006). Pure rotational excitation rate coefficients for $\mathrm{SiO}$ in collision with para- $\mathrm{H}_{2}(j=0)$ were calculated by Turner et al. (1992) in the temperature range $20-300 \mathrm{~K}$ for rotational levels up to $j=20$. The latter rate coefficients were calculated within the coupled-states approximation using a $\mathrm{SiO}-\mathrm{He}$ potential energy surface (PES) that was obtained from an electron gas model (Bieniek \& Green 1981). Although the coupled-states approximation may provide reliable results at moderate to high temperatures, the electron gas model is known to induce large inaccuracies in the PES and hence in the leading rate coefficients 
(Thomas et al. 1980). Moreover, the use of a SiO-He PES to approximate the $\mathrm{SiO}-\mathrm{H}_{2}$ colliding system may also be a source of significant errors given the results obtained for systems such as $\mathrm{CO}$ (Wernli et al. 2006) and $\mathrm{H}_{2} \mathrm{O}$ (Phillips et al. 1996), for which both collision partners were considered.

The goal of the present work is to provide accurate rotational excitation rate coefficients for the $\mathrm{SiO}-\mathrm{He}$ colliding system by means of highly correlated ab initio methods to compute the PES and close-coupling (CC) quantum scattering calculations. Since $\mathrm{H}_{2}$ is the main colliding partner within the astrophysical regions of interest, the SiO-He PES is also employed to provide estimated values of $\mathrm{SiO}-\mathrm{H}_{2}$ collisional rate coefficients. Comparison with the results of Turner et al. (1992) obtained in the same way serves to probe the sensitivity of collisional rate coefficients on the description of the PES. As it will be shown below, quite important discrepancies are found between the two sets of rate coefficients. Hence the present results are thought to represent an important improvement and should be relevant to the modelling of $\mathrm{SiO}$ line emission until the $\mathrm{SiO}-\mathrm{H}_{2}$ colliding system is considered explicitly. This work represents a first step of a thorough theoretical study of ro-vibrational excitation rate coefficients of $\mathrm{SiO}$ by collision with $\mathrm{He}$ and, in the near future, with $\mathrm{H}_{2}$. This is motivated by the perspective of future observations of $\mathrm{SiO}$ line emission by the Herschel Space Observatory, which will acquire data from far-IR to submillimeter domains with both high resolution and sensitivity from a variety of astrophysical regions.

The paper is organised as follows. Details on the ab initio calculations, fitting procedure, and features of the resulting $\mathrm{SiO}-$ He PES are given in Sect. 2. The collisions dynamics calculations are described in Sect. 3. Section 4 is devoted to the results obtained for the $\mathrm{SiO}-\mathrm{He}$ collisional cross sections and rate coefficients, and the approximated $\mathrm{SiO}-\mathrm{H}_{2}$ rate coefficients are compared to those of Turner et al. (1992).

\section{SiO-He PES}

\subsection{Ab initio calculations}

The two interacting species are taken in their ground electronic states $\mathrm{SiO}\left(X^{1} \Sigma^{+}\right)$and $\mathrm{He}\left({ }^{1} \mathrm{~S}\right)$. The $\mathrm{SiO}-\mathrm{He}$ interaction potential is expressed in Jacobi coordinates $(R, r, \theta)$ where $R$ is the distance between the $\mathrm{SiO}$ centre of mass and the He atom, $r$ is the $\mathrm{SiO}$ bond length, and $\theta$ is the angle between the two distance vectors oriented such that $\theta=0^{\circ}$ holds for He approaching the $\mathrm{O}$-side of the $\mathrm{SiO}$ molecule. In the present work the $\mathrm{SiO}$ bond length is held fixed at its equilibrium value $r_{\mathrm{eq}}=2.853 \mathrm{bohr}$ (Huber \& Herzberg 1979). A proper description of the van der Waals interaction potential for the $\mathrm{SiO}-\mathrm{He}$ closed-shell system demands the use of highly correlated electronic calculations. This is needed to properly describe the balance between the short-range repulsion and the attractive long-range dispersion energies. To this aim we performed ab initio calculations in the supermolecular approach using the coupled-cluster method at the RCCSD(T) level of theory (Watts et al. 1993). The basis sets employed to describe the $\mathrm{Si}, \mathrm{O}$, and $\mathrm{He}$ atoms consisted of the standard aug-cc-pVQZ basis sets (Dunning 1989; Kendall et al. 1992; Woon \& Dunning 1993, 1994). Added to this set, the [3s $2 \mathrm{p} 2 \mathrm{~d} 1 \mathrm{f} 1 \mathrm{~g}]$ bond functions optimised by Cybulski \& Toczylowski (1999) were placed at the midpoint between the $\mathrm{He}$ atom and the $\mathrm{SiO}$ centre of mass. At each geometry the calculated energy $E_{\mathrm{SiO}-\mathrm{He}}(R, \theta)$ was corrected for the basis set superposition error (BSSE) using the Boys \& Bernardi (1970) counterpoise procedure:

$V(R, \theta)=E_{\mathrm{SiO}-\mathrm{He}}(R, \theta)-E_{\mathrm{SiO}}(R, \theta)-E_{\mathrm{He}}(R, \theta)$, where the $\mathrm{SiO}$ and $\mathrm{He}$ subsystems are computed with the $\mathrm{SiO}-\mathrm{He}$ basis set. Additional calculations of $E_{\mathrm{SiO}-\mathrm{He}}(R, \theta)$ at a large number of $(R, \theta)$ geometries were performed using the multiconfigurational CASSCF approach (Werner \& Knowles 1985; Knowles $\&$ Werner 1985). The weight associated with the closed-shell electronic configuration used as a reference in the coupledcluster calculations was found to be by far the dominant one (the weight at least equals 0.94 for all studied geometries). Under such circumstances, and owing to the rather extended basis sets used in the present work, the coupled-cluster approach is expected to yield reliable results for the $\mathrm{SiO}-\mathrm{He}$ interaction potential. All ab initio calculations have been carried out with the MOLPRO (Werner et al. 2003) suite of programs.

Using the computational scheme described above, a total of 640 geometries were computed for the $\mathrm{SiO}-\mathrm{He}$ system. The radial scattering coordinate $R$ ranged from 4 to 16 bohr by steps of $0.25 \mathrm{bohr}$, and the angle $\theta$ varied from $0^{\circ}$ to $180^{\circ}$ by steps of $15^{\circ}$. At a short atom-diatom separation, additional geometries were computed in such a way that the interaction energy becomes at least $3500 \mathrm{~cm}^{-1}$ at any angle.

\subsection{Fitting procedure}

An analytic representation of the two-dimensional interaction potential $V(R, \theta)$, suitable for use in dynamics calculations, was constructed following the method developed by Werner et al. (1989). At each computed angle $\theta_{n}$ the $R$-dependent potentials $V\left(R, \theta_{n}\right)$ were fitted to the form:

$$
\begin{aligned}
B_{n}(R)= & \exp \left(-a_{n} R\right) \cdot\left[\sum_{i=0}^{3} b_{n}^{(i)} R^{i}\right] \\
& -\frac{1}{2}[1+\tanh (R)] \cdot\left[\sum_{j=6,8} c_{n}^{(j)} R^{-j}\right],
\end{aligned}
$$

where the first and second parts of Eq. (2) hold for the shortand long-range parts of the potential, respectively. For each of the angles $\theta_{n}$, a set of seven parameters was determined from the calculated interaction energies $V\left(R, \theta_{n}\right)$, leading to a global root-mean-square deviation between the fit and the whole set of ab initio points of $0.85 \mathrm{~cm}^{-1}$ (the mean relative error is $0.07 \%$ with a largest deviation of $0.54 \%$ ). The one-dimensional functions $B_{n}(R)$ were then used to express the analytic interaction potential $V(R, \theta)$ as an expansion into a basis of Legendre polynomials up to $\lambda_{\max }=12$ :

$$
V(R, \theta)=\sum_{\lambda=0}^{L-1} V_{\lambda}(R) P_{\lambda}(\cos \theta),
$$

where $L=13$ is the number of computed angle values and $V(R)$ is a vector column obtained from $\boldsymbol{V}=\mathbf{P}^{-1} \boldsymbol{B}$, with matrix elements of $\mathbf{P}$ given by $P_{n \lambda}=P_{\lambda}\left(\cos \theta_{n}\right)$.

As a check of our fitting procedure, the ab initio points were interpolated along the angular coordinate to generate an additional PES expressed onto 25 angle values. The latter PES was then fitted using the polynomial expansion of Eq. (3) up to $\lambda_{\max }=24$. For $\lambda<8$ the resulting anisotropic terms $V_{\lambda}(R)$ were found to be almost identical to those obtained from the ab initio PES defined onto 13 angle values. For the higher order components, discrepancies appear only at short $R$ values, i.e. within classically forbidden regions of the PES. Such a check pointed out that our ab initio PES defined onto 13 angle values 


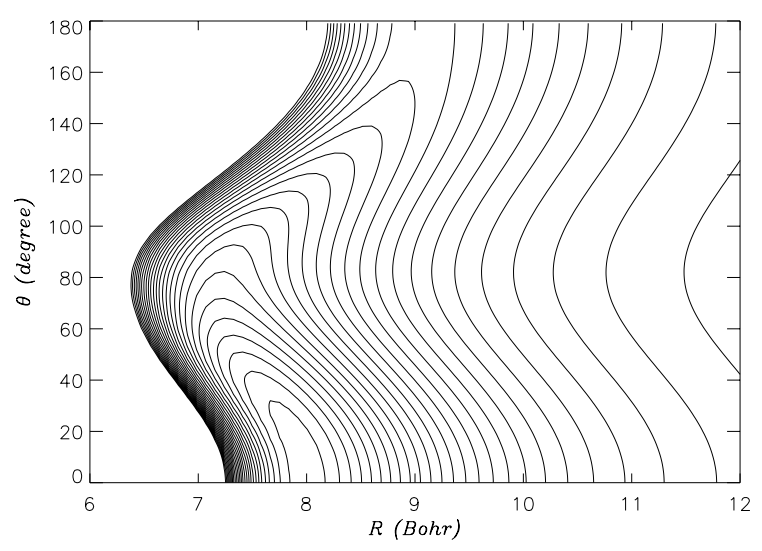

Fig. 1. Contour plot of the analytical ground PES of the SiO-He system obtained from the present ab initio calculations, as a function of Jacobi coordinates $R$ and $\theta$. The $\mathrm{SiO}$ bond length is held fixed to $r_{\mathrm{eq}}=2.853$ bohr. Contours are drawn in steps of $1 \mathrm{~cm}^{-1}$ with a maximum energy of $0 \mathrm{~cm}^{-1}$.

was sufficient to properly describe the anisotropy of the $\mathrm{SiO}-\mathrm{He}$ interaction potential.

\subsection{Features of the PES}

A contour plot of the analytical PES $V(R, \theta)$ is shown in Fig. 1 as a function of Jacobi coordinates $R$ and $\theta$ for the $\mathrm{SiO}$ bond length kept fixed at $r_{\text {eq }}=2.853 \mathrm{bohr}$. The interaction potential is found to be highly anisotropic, with both long- and short-range interactions weaker for the perpendicular approach $\left(\theta=90^{\circ}\right)$ of the $\mathrm{He}$ atom toward the $\mathrm{SiO}$ molecule than for collinear approaches $\left(\theta=0^{\circ}\right.$ and $\left.180^{\circ}\right)$. The linear He-OSi geometry $\left(\theta=0^{\circ}\right)$ is found to be favoured over the linear $\mathrm{He}-\mathrm{SiO}\left(\theta=180^{\circ}\right)$, due to weaker short-range interactions as $\mathrm{He}$ approaches the $\mathrm{O}$-side of the $\mathrm{SiO}$ molecule. Such features result in a global minimum for the PES at the linear He-OSi geometry (and $R=7.993 \mathrm{bohr}$ ) and an associated well depth $\epsilon=-26.596 \mathrm{~cm}^{-1}$.

The main features of the PES described above are consistent with what one might expect from the features associated with the polarizability of the $\mathrm{SiO}$ molecule $\left(\alpha_{\|}>\alpha_{\perp}\right)$ and with the polarizabilities of the $\mathrm{Si}$ and $\mathrm{O}$ atoms $\left(\alpha_{\mathrm{Si}} \gg \alpha_{\mathrm{O}}\right)$. As a check, the $\mathrm{SiO}-\mathrm{He} \mathrm{PES}$ was also computed by means of an empirical model developed by Pirani et al. (2001) and further extended by Bartolomei et al. (2006) to account for heteronuclear diatoms. The resulting PES $^{1}$ qualitatively reproduces the main features of our ab initio PES. The empirical model told us that the location of the global minimum at $\theta=0^{\circ}$ is mainly due to a combination of dipole-induced dipole and (dipole, quadrupole)-induced dipole interactions that favour the linear He-OSi geometry over the other ones. From the empirical PES a global minimum of a depth $\epsilon=-23.153 \mathrm{~cm}^{-1}$ is found at $\theta=0^{\circ}$ (and $R=7.667 \mathrm{bohr}$ ), which is in quite good agreement with the results obtained from our ab initio PES.

1 The empirical PES accounts for short-range repulsion, dispersion, dipole-induced dipole and (dipole, quadrupole)-induced dipole interactions. The parameters that were used to build the PES are: the atomic polarizabilities $\alpha_{\mathrm{He}}=1.41 a_{0}^{3}$ (Olney et al. 1997), $\alpha_{\mathrm{Si}}=36.32 a_{0}^{3}$ (Reinsch $\&$ Meyer 1976), and $\alpha_{\mathrm{O}}=5.20 a_{0}^{3}$ (Werner \& Meyer 1976); the dipole moment $\mu=-1.22 e a_{0}$ (Ramonda et al. 1970) and quadrupole moment $Q=-3.39 e a_{0}^{2}$ (Honerjäger \& Tischer 1974) of the $\mathrm{SiO}$ molecule; and the molecular bond polarizabilities $\alpha_{\|}=41.62 a_{0}^{3}$ and $\alpha_{\perp}=37.39 a_{0}^{3}$ of the $\mathrm{SiO}$ molecule were obtained from an empirical method (Bartolomei et al. 2006).

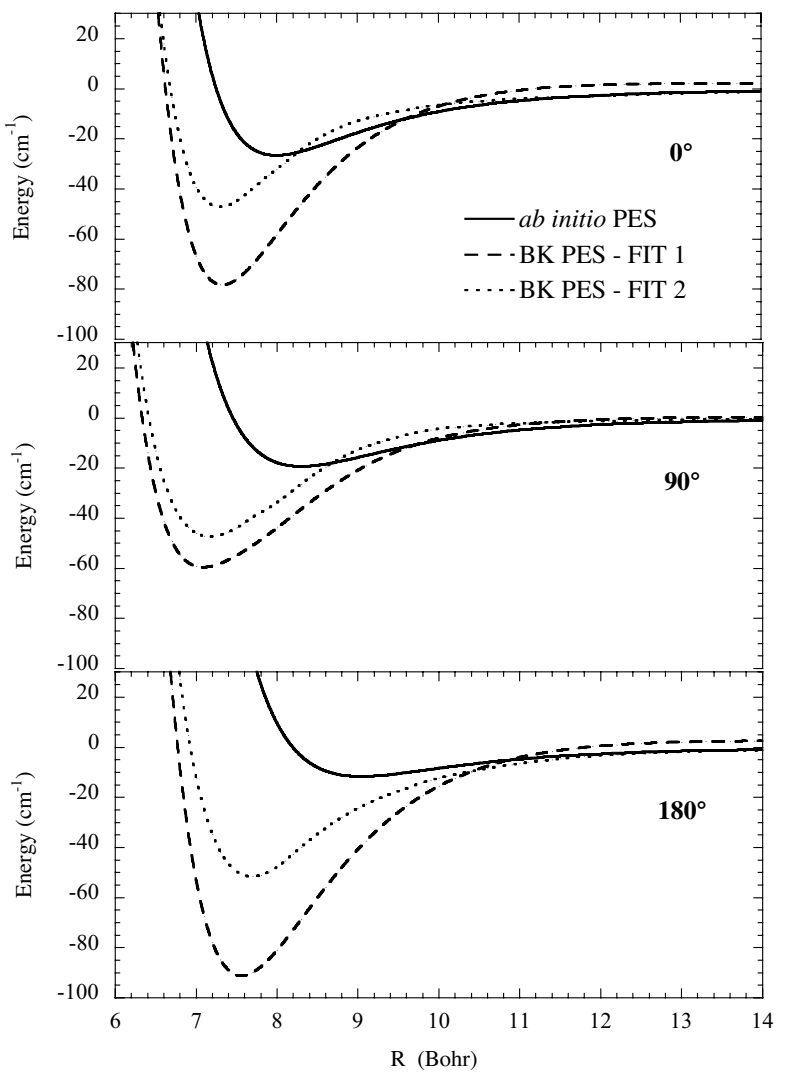

Fig. 2. Cuts of the analytical SiO-He PES at given values of the Jacobi angle. The ab initio PES obtained in the present work is compared to the PESs computed by Bieniek \& Green (1981) (BK PES-FIT1) and by Sisak \& Secrest (1991) (BK PES-FIT2).

A comparison can be made with the SiO-He PES computed by Bieniek \& Green (1981) using the electron-gas method (Gordon \& Kim 1972) and an analytic form for long-range interactions. The PES was originally fitted (FIT1) with the aim of calculating ro-vibrational excitation rate coefficients at high temperatures and was not expressed in terms of a Legendre polynomial expansion. A second analytical fit (FIT2) to the energy points of Bieniek and Green (BK) was obtained by Sisak \& Secrest (1991) to compute rotational excitation cross sections at low collision energies, using a Legendre polynomial expansion up to $\lambda_{\max }=6$. The rotational excitation rate coefficients calculated by Turner et al. (1992) were obtained from a third fit, with an interpolation procedure for the angular coordinate that allowed them to express the PES in terms of a Legendre polynomial expansion up to $\lambda_{\max }=12$. Details on the latter PES were not published, but it is believed to be rather similar to FIT2. We report in Fig. 2 cuts of the two available BK PESs at given values of the Jacobi angle $\theta$, together with the result of the present ab initio calculations. It is readily seen that both BK PESs are markedly different from the ab initio one. At whatever geometry, they show potential wells deeper by at least a factor of 2 and shifted to shorter $R$ values compared to the ab initio PES. Moreover, the global minima associated with the PESs FIT1 and FIT2 are found at $\theta=180^{\circ}\left(\epsilon=-91.193 \mathrm{~cm}^{-1}\right)$ and $\theta=54^{\circ}$ $\left(\epsilon=-57.875 \mathrm{~cm}^{-1}\right)$, respectively, in direct contrast to the global minimum found at $\theta=0^{\circ}$ for both the ab initio and empirical PESs.

To assess the main features of the SiO-He PES likely to influence the scattering processes we report in Fig. 3 the first four radial terms $V_{\lambda}(R)$ of Eq. (3) associated with the ab initio PES 


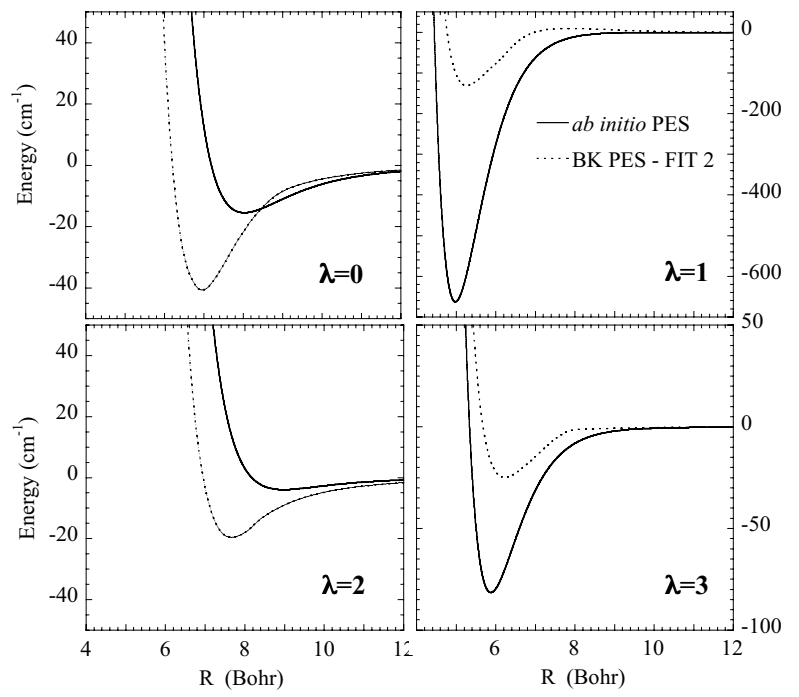

Fig. 3. Comparison of the first four $V_{\lambda}(R)$ radial terms associated with the $\mathrm{SiO}-\mathrm{He}$ PESs obtained from the present ab initio calculations and the analytic fit (FIT2) of Sisak \& Secrest (1991) to the energy points computed by Bieniek \& Green (1981).

together with the results obtained by Sisak \& Secrest (1991). One has to recall that the isotropic component $\lambda=0$ only contributes to the elastic scattering process. Moreover, each $\left(j, j^{\prime}\right)$ pair of states characterised by even (or odd) $\Delta j=\left|j-j^{\prime}\right|$ values are directly coupled by even (or odd) $V_{\lambda}(R)$ terms which verify $\left|j-j^{\prime}\right| \leq \lambda \leq j+j^{\prime}$. For each $\Delta j$ inelastic transition, the remaining $V_{\lambda}(R)$ terms contribute through indirect coupling with other rotational states. The isotropic term $V_{0}$ depicted in Fig. 3 is found to be much less attractive for the ab initio PES compared to the BK PES, with a repulsive wall displaced toward larger $R$ values. A similar trend is found for the anisotropic $V_{2}$ term. By contrast, the anisotropic terms $V_{1}$ and $V_{3}$ are found much more attractive for the ab initio PES than for the BK PES, with a repulsive wall slightly displaced toward shorter $R$ values. The higher order components $4 \leq \lambda \leq 12$ associated with the ab initio PES are mainly repulsive terms and their magnitudes rapidly decrease as $\lambda$ increases. No significant differences are found between the ab initio and BK PESs for the components $4 \leq \lambda \leq 6$. However, the large differences found between the two PESs on the isotropic component $\lambda=0$ and the main anisotropic components $1 \leq \lambda \leq 3$ are expected to yield inelastic cross sections, and hence rate coefficients, that are significantly different. This will be discussed in the next sections.

\section{Collision dynamics calculations}

The analytic SiO-He interaction potential fitted to the ab initio points was used to perform $\mathrm{CC}$ calculations of the rotational excitation and de-excitation cross sections using the MOLSCAT code (Hutson \& Green 1994). The ${ }^{28} \mathrm{Si}^{16} \mathrm{O}-{ }^{4} \mathrm{He}$ system was considered $\left(\mu_{\mathrm{SiO}-\mathrm{He}}=3.66865811\right.$ a.m.u.). The rotational energy levels were described using a set of Dunham ro-vibrational constants (Lovas et al. 1981) that were fitted to experimental line frequencies. The coupled-channel Schrödinger equations were solved using the diabatic modified log-derivative method of Manolopoulos (1986). Terms up to $\lambda_{\max }=11$ were retained in the Legendre polynomial expansion of the potential defined by Eq. (3), and a 13 point Gauss-Legendre quadrature was used to obtain the radial expansion coefficients $V_{\lambda}(R)$. The minimum propagation distance was held fixed at about 5 bohr for low energies (typically $100 \mathrm{~cm}^{-1}$ ), whereas an automatic search of this parameter was used at higher energies with the criterion that the wavefunction amplitude should be less than $10^{-9}$ in all channels at the starting point. The minimum value of the maximum propagation distance was set to $40 \mathrm{bohr}$, and this value was increased when necessary according to the location of the outer classical turning point on the centrifugal energy barrier. The maximum value of the total angular momentum $J$ used in the calculations was chosen according to a convergence criterion of $0.005 \AA^{2}$ on the inelastic cross sections value. CC calculations were carried out for total energies ranging from the first excited rotational energy level up to $3000 \mathrm{~cm}^{-1}$. Owing to the relatively low value of the $\mathrm{SiO}$ rotational constant $\left(B_{v=0}=0.724 \mathrm{~cm}^{-1}\right)$, a large number of rotational levels had to be included in the basis set to ensure convergence of the inelastic cross sections. The size of the basis set varied from $j_{\max }=8$ up to $j_{\max }=32$ according to the range of energies studied. Test calculations were performed over the whole range of energies to check that the chosen propagation parameters and basis set size ensure an accuracy of about $1 \%$ for the inelastic cross sections values.

Using the computational scheme described above, inelastic cross sections associated with a transition from an initial rotational state $j$ to a final state $j^{\prime}$ were obtained at each kinetic energy $E_{k}$ from (Arthurs \& Dalgarno 1963):

$$
\begin{aligned}
\sigma_{j \rightarrow j^{\prime}}\left(E_{k}\right)= & \frac{\pi}{(2 j+1) k_{j}^{2}} \sum_{J=0}^{\infty}(2 J+1) \\
& \times \sum_{l=|J-j|}^{|J+j|} \sum_{l^{\prime}=\left|J-j^{\prime}\right|}^{\left|J+j^{\prime}\right|}\left|\delta_{j j^{\prime}} \delta_{l l^{\prime}}-S_{j^{\prime} l^{\prime} ; j l}^{J}(E)\right|^{2},
\end{aligned}
$$

where $J$ and $l$ are the total and orbital angular momentum quantum numbers respectively, $E$ is the total energy, and $E_{k}=$ $\hbar^{2} k_{j}^{2} / 2 \mu_{\mathrm{SiO}-\mathrm{He}}=E-\epsilon_{j}$, where $\epsilon_{j}$ is the rotational energy level of the initial $j$ state. From the rotational excitation cross sections $\sigma_{j \rightarrow j^{\prime}}\left(E_{k}\right)$, one obtains the rotational excitation rate coefficient at a temperature $T$ by performing a Boltzmann thermal average of the cross sections:

$$
\begin{aligned}
k_{j \rightarrow j^{\prime}}(T)= & \left(\frac{8 k_{\mathrm{B}} T}{\pi \mu_{\mathrm{SiO}-\mathrm{He}}}\right)^{\frac{1}{2}} \frac{1}{\left(k_{\mathrm{B}} T\right)^{2}} \\
& \times \int_{0}^{\infty} \sigma_{j \rightarrow j^{\prime}}\left(E_{k}\right) \exp \left(-E_{k} / k_{\mathrm{B}} T\right) E_{k} \mathrm{~d} E_{k},
\end{aligned}
$$

where $k_{\mathrm{B}}$ is the Boltzmann constant. The numerical integration over the collision energies was performed using a Simpson rule. Care was taken to span the energy range with a step size low enough to account for resonance structures in the cross sections. To this aim the energy step size was progressively increased from 0.05 to $1 \mathrm{~cm}^{-1}$ for total energies from 1.4 up to $850 \mathrm{~cm}^{-1}$, from 2 to $50 \mathrm{~cm}^{-1}$ for energies up to $2000 \mathrm{~cm}^{-1}$, and then set equal to $500 \mathrm{~cm}^{-1}$ for energies up to $3000 \mathrm{~cm}^{-1}$. Such an energy range was found large enough to calculate rotational excitation rate coefficients up to $300 \mathrm{~K}$ for rotational levels up to $j=26$, the highest energy point for each transition being at least at the threshold energy plus $12 k T$. The rotational excitation rate coefficients $k_{j \rightarrow j^{\prime}}(T)$ obtained from Eq. (5) can then be used to compute the de-excitation rate coefficients $k_{j^{\prime} \rightarrow j}(T)$ from the detailed balance:

$k_{j^{\prime} \rightarrow j}(T)=\frac{(2 j+1)}{\left(2 j^{\prime}+1\right)} \exp \left[-\left(\epsilon_{j}-\epsilon_{j^{\prime}}\right) / k_{\mathrm{B}} T\right] k_{j \rightarrow j^{\prime}}(T)$. 

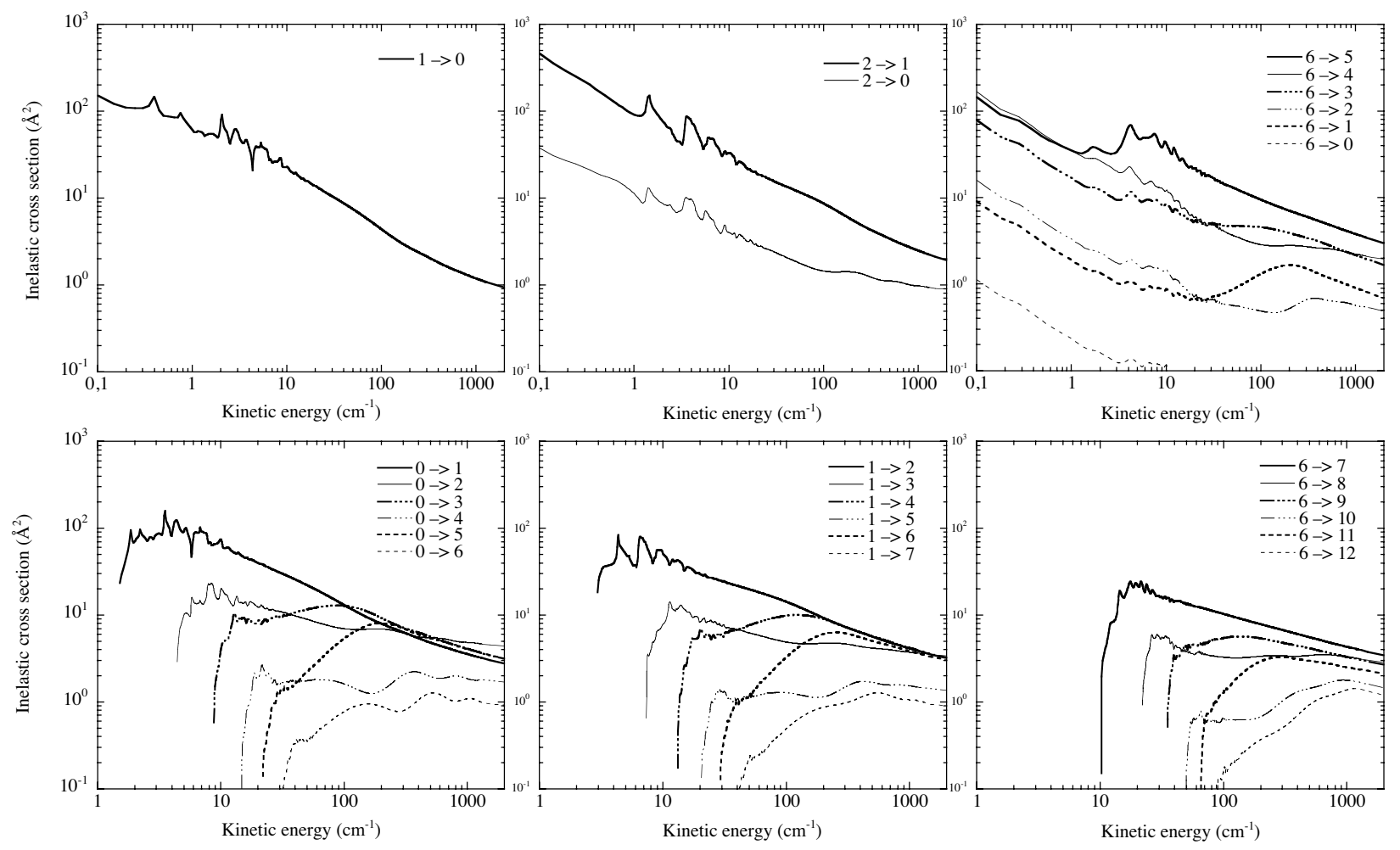

Fig. 4. Rotational de-excitation and excitation cross sections (upper and lower panel, respectively) of the SiO molecule in collision with He as a function of the relative kinetic energy. A logarithmic scale is used for both axes. Bold lines: odd $\Delta j=\left|j-j^{\prime}\right|$ inelastic transitions; thin lines: even $\Delta j=\left|j-j^{\prime}\right|$ inelastic transitions.

The de-excitation rate coefficients obtained from the deexcitation cross sections by means of Eq. (5) were used as a check of our numerical integration procedure.

\section{Results and discussion}

\subsection{Cross sections}

We report in Fig. 4 the de-excitation and excitation cross sections obtained from the present $\mathrm{CC}$ calculations for a few selected rotational levels of the $\mathrm{SiO}$ molecule. The de-excitation cross sections are almost decreasing functions of collision energies $E_{k}$. Interestingly, the present SiO-He PES leads to $\Delta j=1$ and $\Delta j=2$ de-excitation cross sections that behave roughly like $E_{k}^{-1 / 2}$ and $E_{k}^{-1 / 6}$, respectively, at high collision energies. For collision energies below $30 \mathrm{~cm}^{-1}$ many resonances are found in the de-excitation cross sections. This relates to the presence of an attractive potential well, of a depth of $\epsilon=-27 \mathrm{~cm}^{-1}$ at the linear $\mathrm{He}-\mathrm{OSi}$ geometry, which allows the $\mathrm{He}$ atom to be temporarily trapped into the well and hence quasibound states to be formed before the complex dissociates (Smith et al. 1979; Christoffel \& Bowman 1983). Quasibound states may arise from tunneling through the centrifugal energy barrier (shape resonances) or they may form through excitation of the molecule to an asymptotically closed channel where the complex is still temporarily trapped before it de-excites to open channels (Feshbach resonances). Owing to the rather small energy spacing between the $\mathrm{SiO}$ rotational levels with respect to the potential well depth, both types of resonance may be found in the same energy range. This was confirmed on selected transitions by strong changes in the resonance structure when the basis set size or the rotational energy spacing are modified in the scattering calculations.
The de-excitation cross sections shown in Fig. 4 exhibit further interesting features that have important consequences on the magnitude of rotational de-excitation rate coefficients: $(i)$ for a given initial $j$ state the de-excitation cross sections decrease on increasing $\Delta j$, with propensity rules giving rise to odd $\Delta j$ cross sections larger than even $\Delta j$ cross sections; ( $i i$ ) for a given $\Delta j$ the de-excitation cross sections increase with $j$, the effect being more pronounced for low $j$ values. The features described above follow the trend suggested by the anisotropic terms $V_{\lambda}$, which couple directly each $\left(j, j^{\prime}\right)$ pair of states: $(i)$ for a given initial $j$ state the order $\lambda$ increases with $\Delta j$ and the magnitude of $V_{\lambda}$ terms decreases on increasing $\lambda$ for the classically allowed regions of the PES. This may explain the rapidly diminished coupling between the rotational states as $\Delta j$ increases. The propensity rule observed for odd and even $\Delta j$ cross sections is probably due to the large well depths associated with the $V_{1}$ and $V_{3}$ terms (see Fig. 3) that favour odd $\Delta j$ transitions over even ones. Interestingly, the propensity rule is still observed up to $\Delta j=8$, i.e., for rotational transitions where the $V_{1}$ and $V_{3}$ terms only contribute through indirect coupling with other rotational states. (ii) For a given value of $\Delta j$ the number of $V_{\lambda}$ terms involved increases with $j$, which may yield a stronger coupling between the rotational states. The latter effect becomes rapidly saturated with $j$ due to the decreasing magnitude of $V_{\lambda}$ terms as $\lambda$ increases.

The features shown by excitation cross sections in Fig. 4 can be understood from the detailed balance:

$\sigma_{j \rightarrow j^{\prime}}\left(E_{k}\right)=\frac{\left(E-\epsilon_{j^{\prime}}\right)}{\left(E-\epsilon_{j}\right)} \frac{\left(2 j^{\prime}+1\right)}{(2 j+1)} \sigma_{j^{\prime} \rightarrow j}\left(E_{k}\right)$,

which relates excitation and de-excitation cross sections. For the relatively low $j$ and $\Delta j$ values considered in Fig. 4 the energy factor $\left(E-\epsilon_{j^{\prime}}\right) /\left(E-\epsilon_{j}\right)$, responsible for the shape of excitation 


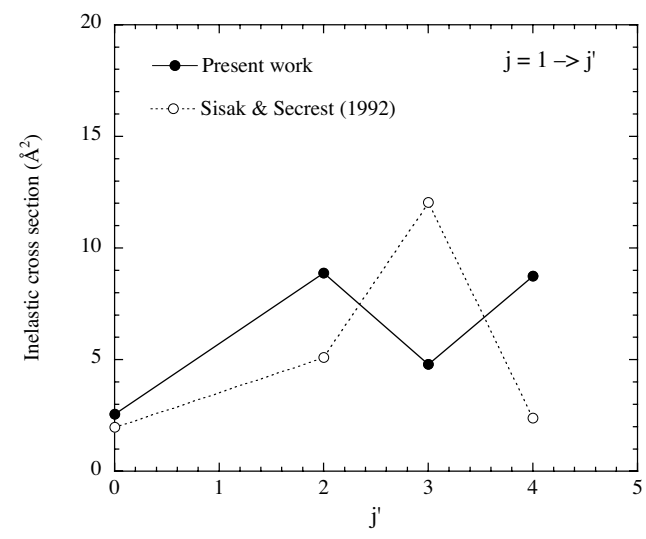

Fig. 5. Rotational (de)excitation cross sections of the $\mathrm{SiO}$ molecule in collision with $\mathrm{He}$ for the $j=1 \rightarrow j^{\prime}=0-4$ inelastic transitions at the total energy $E=218.0 \mathrm{~cm}^{-1}$. Results obtained from the present work and CC calculations of Sisak \& Secrest (1992) using the PES of Bieniek \& Green (1981).

cross sections above energy thresholds, rapidly goes to 1 with increasing energy. Hence the main contribution to the observed features of excitation cross sections with respect to de-excitation ones comes from the degeneracy factor $\left(2 j^{\prime}+1\right) /(2 j+1)$. For a given initial $j$ state it increases continuously with $\Delta j$, while for a given $\Delta j$ it decreases with $j$.

The present results have been compared to inelastic cross section values reported by Sisak \& Secrest $(1991,1992)$ for selected transitions and given values of the total energy. The (de)excitation cross sections were computed by means of $\mathrm{CC}$ calculations using the PES of Bieniek \& Green (1981). For collision energies above the well depths of both PESs, the comparison points out that the present ab initio $\mathrm{SiO}-\mathrm{He}$ interaction potential leads to greater odd $\Delta j$ cross sections than the BK PES (see Fig. 5). The enhancement is about $30 \%$ for the $1 \rightarrow 0$ transition, $70 \%$ for the $1 \rightarrow 2$ transition, and up to a factor of 3.5 for the $1 \rightarrow 4$ and $3 \rightarrow 0$ transitions (not shown in Fig. 5). By contrast, a decrease of the cross section by a factor close to 2.5 is observed for the $1 \rightarrow 3$ transition corresponding to an even $\Delta j$ value. Consequently the two PESs yield opposite propensity rules for even and odd $\Delta j$ cross sections. In the present case the propensity rule $\sigma$ (odd $\Delta j)>\sigma($ even $\Delta j)$ arises from the large asymmetry of the ab initio $\mathrm{SiO}-\mathrm{He}$ interaction potential, leading to odd $V_{1}$ and $V_{3}$ anisotropic terms characterised by deep well depths. It is worth noticing that such an unusual propensity rule for rotational excitation cross sections has been already discussed by McCurdy \& Miller (1977) in terms of model potentials, and that it has been observed experimentally (Carty et al. 2004) as well as from theoretical calculations (Cecchi-Pestellini et al. 2002) for the CO-He collision system.

\subsection{Rate coefficients}

The rotational excitation and de-excitation rate coefficients of $\mathrm{SiO}(j)$ in collision with He have been computed for rotational levels up to $j=26$ and kinetic temperatures up to $300 \mathrm{~K}$ through a Boltzmann thermal average of inelastic cross sections (Eq. (5)). We report in Fig. 6 the de-excitation rate coefficients as a function of $\Delta j=j-j^{\prime}$ for selected transitions and temperatures. It is seen that: $(i)$ for a given initial $j$ state the de-excitation rate coefficients decrease with increasing $\Delta j$, with propensity rules giving rise to odd $\Delta j$ rate coefficients larger than even $\Delta j$ ones; ( $i i)$ for a given $\Delta j$ the de-excitation rate coefficients increase with $j$, the effect being more pronounced for low $j$ values. The features described above were expected from the results obtained for the deexcitation cross sections and discussed in the previous section.

Figure 6 also shows the variation of selected de-excitation rate coefficients with temperature. As can be seen the rate coefficients associated with a given value of $\Delta j$ evolve in a similar fashion with temperature. This comes from the dominant contribution of the first anisotropic term $V_{\lambda}$, with $\lambda=\Delta j$, specific to each $\Delta j$ inelastic transition. Furthermore, $\Delta j=1$ rate coefficients are almost constant over the whole temperature range. This is a direct consequence of the variation close to $E_{k}^{-1 / 2}$ of the associated de-excitation cross sections (the integral of Eq. (5) behaves like $T^{3 / 2}$ in such a case). The rate coefficient corresponding to the $1 \rightarrow 0$ transition deviates from this rule. It rather behaves like $T^{4 / 3}$ due to the steeper variation of its associated deexcitation cross section, close to $E_{k}^{-2 / 3}$. For $\Delta j=2$, the variation close to $E_{k}^{-1 / 6}$ of de-excitation cross sections at high collision energies produces an increase in the rate coefficient close to $T^{1 / 3}$ above $100 \mathrm{~K}$. The complete set of (de)excitation rate coefficients is available as on-line material from the BASECOL web site ${ }^{2}$.

\subsection{Comparison with previous results}

In the circumstellar environment of evolved stars and shocked regions of the interstellar medium, the relatively large abundance of $\mathrm{H}_{2}$ makes this species the main colliding partner for other molecules. The collisional rate coefficients corresponding to molecules in collision with $\mathrm{H}_{2}$ are thus needed to infer both the molecular abundances and physical conditions in these regions from the observed molecular spectra. Unless these rate coefficients are available, it is generally assumed (Schöier et al. 2005) that rate coefficients with $\mathrm{He}$ as a collision partner are likely to provide a first estimate of rate coefficients with para$\mathrm{H}_{2}(j=0)$. The underlying approximation is to consider identical cross section values for the two colliding systems, and then apply a scaling factor to the rate coefficients to correct for the associated different reduced masses. For a given molecule $\mathrm{X}$, the scaling factor is $\left(\mu_{\mathrm{X}-\mathrm{He}} / \mu_{\mathrm{X}-\mathrm{H}_{2}}\right)^{1 / 2}$ (see Eq. (5)) and equals about 1.4 if the molecule $\mathrm{X}$ is much heavier than $\mathrm{He}$ and $\mathrm{H}_{2}$. However, results on systems such as CO (Wernli et al. 2006) and $\mathrm{H}_{2} \mathrm{O}$ (Phillips et al. 1996), for which both collision partners were considered, pointed out that rate coefficients associated with p$\mathrm{H}_{2}(j=0)$ were within a factor of 1-3 larger than those with He depending on the selected transition. Another widely used approximation is to consider the interaction potential between a given molecule $\mathrm{X}$ and He identical to the one associated with $\mathrm{p}-\mathrm{H}_{2}(j=0)$, and then perform scattering calculations using the reduced mass $\mu_{\mathrm{X}-\mathrm{H}_{2}}$ to get the associated rate coefficients. This was indeed the case for the $\mathrm{SiO}-\mathrm{H}_{2}$ rate coefficients calculated by Turner et al. (1992) and currently used in radiative transfer modelling of interstellar regions. Thus, to probe the effect of the new $\mathrm{SiO}-\mathrm{He}$ interaction potential on collisional rate coefficients, we employed the same approximation as Turner et al. (1992) to compute the $\mathrm{SiO}-\mathrm{H}_{2}$ rate coefficients.

The scattering calculations were performed by means of the CC method. Similar basis sets and energy grid spacing as used before were employed for total energies up to $350 \mathrm{~cm}^{-1}$. For energies above $500 \mathrm{~cm}^{-1}$ we took advantage of the smooth variation of collisional cross sections (see Fig. 4) to use an energy grid spacing of $500 \mathrm{~cm}^{-1}$ up to $3000 \mathrm{~cm}^{-1}$. This allowed the determination of rate coefficients for the twenty first rotational

\footnotetext{
${ }^{2}$ amdpo.obspm.fr/basecol/
} 

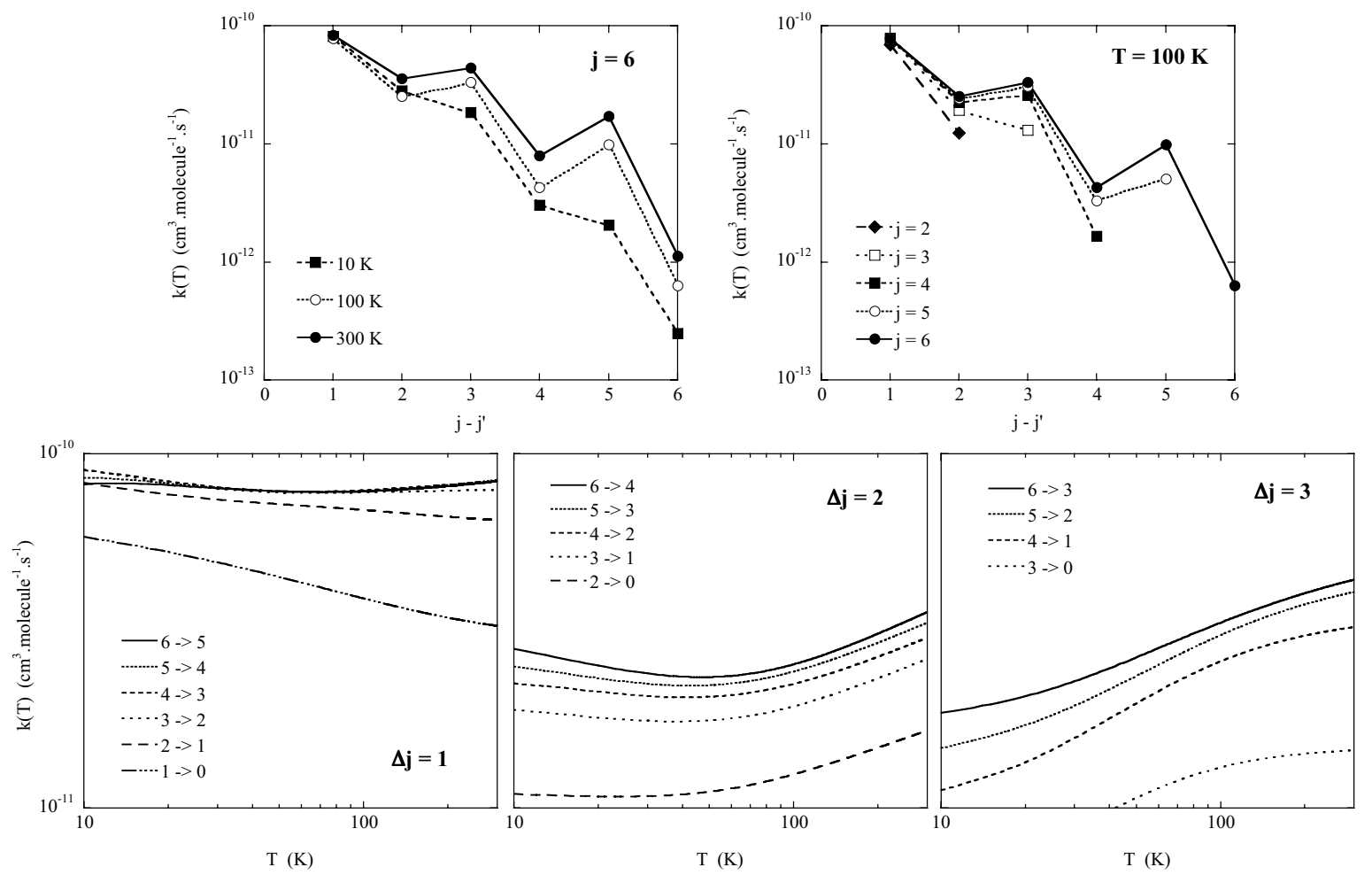

Fig. 6. Rotational de-excitation rate coefficients of the $\mathrm{SiO}$ molecule in collision with He. Upper-left panel: rate coefficients for $\mathrm{SiO}(j=6)$ as a function of $\Delta j=j-j^{\prime}$ for several temperatures; upper-right panel: rate coefficients for $\operatorname{SiO}(j=2-6)$ and $T=100 \mathrm{~K}$ as a function of $\Delta j$; lower panel: rate coefficients for $\mathrm{SiO}(j=1-6)$ as a function of temperature for $\Delta j=1-3$.

energy levels up to $300 \mathrm{~K}$. The dominant rate coefficients obtained from the two approximations described above are shown in Fig. 7, together with the results of Turner et al. (1992). As can be seen first, the scaled $\mathrm{SiO}-\mathrm{He}$ rate coefficients compare rather well with our estimated values for the $\mathrm{SiO}-\mathrm{H}_{2}$ collision system for low $\Delta j$ transitions, the discrepancies being around $10 \%$ and $30 \%$ at high and low temperatures, respectively. Nevertheless, for higher $\Delta j$ transitions the typical errors rise continuously, up to factors of 2 and 25 for $\Delta j=11$ at high and low temperatures, respectively. This illustrates the role played by the reduced mass accounted for in collision dynamics calculations. On the other hand, the comparison between our rate coefficients and those of Turner et al. (1992) show quite important disagreements even for low $\Delta j$ transitions: $(i)$ for odd $\Delta j$ transitions our rate coefficients are larger than those of Turner et al. (1992) by about a factor of 5, up to an order of magnitude larger for high $j$ levels at low temperatures; (ii) for even $\Delta j$ transitions our rates are smaller than those of Turner et al. (1992) by about a factor of 2.5 whatever the temperature; (iii) for higher $\Delta j$ transitions (not shown here) the discrepancies can rise up to an order of magnitude even at high temperatures; (iv) the two sets of rate coefficients display opposite propensity rules for even and odd $\Delta j$ transitions.

Such large differences may be attributed to several factors, the main one being the distinct $\mathrm{SiO}-\mathrm{He}$ interaction potentials employed in dynamics calculations. Indeed, the $\mathrm{SiO}-\mathrm{He}$ interaction potential of Bieniek \& Green (1981) computed by means of an electron gas method has been found markedly different from the present ab initio SiO-He PES. It is worth mentioning that details of the $\mathrm{SiO}-\mathrm{He} \mathrm{PES}$ resulting from the fitting procedure of Turner et al. (1992) were not published. Nevertheless, the leading rate coefficients and cross section values of Sisak \& Secrest (1991, 1992) yield close differences when compared to our results (see end of Sect. 4.1). Thus the SiO-He PES employed by
Turner et al. (1992) is thought to be almost similar to the one of Sisak \& Secrest $(1991,1992)$ discussed in Sect. 2. At low temperatures, the discrepancies between the two sets of rate coefficients may also be attributed both to the use of the coupled-states approximation in dynamics calculations and to the description of resonance structure in collisional cross sections.

We have to stress that the underlying approximation to the determination of our $\mathrm{SiO}-\mathrm{H}_{2}$ collisional rate coefficients is identical to the one employed by Turner et al. (1992). Since accurate values for $\mathrm{SiO}-\mathrm{H}_{2}$ rate coefficients are still lacking, the values of Turner et al. (1992) have been employed so far for the SiO line emission modelling of many astrophysical regions (see for instance González Delgado et al. (2003); Gibb et al. (2004) for the most recent studies). However, discrepancies as large as a factor of 2.5-5 between two sets of collisional rate coefficients for the main rotational transitions are expected to be relevant in radiative transfer modelling since they should strongly modify the estimated values of column density (Lique et al. 2006). Hence, although the present study focused on the determination of accurate rate coefficients for the $\mathrm{SiO}-\mathrm{He}$ collision system, our estimated values for $\mathrm{SiO}-\mathrm{H}_{2}$ are thought to be the most appropriate for an astrophysical purpose until the $\mathrm{SiO}-\mathrm{H}_{2}$ colliding system be explicitly considered.

\section{Conclusions}

Inelastic cross sections corresponding to pure rotational (de)excitation of $\mathrm{SiO}$ by collision with He have been computed from CC quantum scattering calculations in the energy range 1.4-3000 $\mathrm{cm}^{-1}$, using a rotational basis set extending up to $j_{\max }=32$. This allowed the determination of thermally averaged rate coefficients associated with transitions among the rotational levels of $\mathrm{SiO}$ up to $j=26$ for kinetic temperatures up to $300 \mathrm{~K}$. 

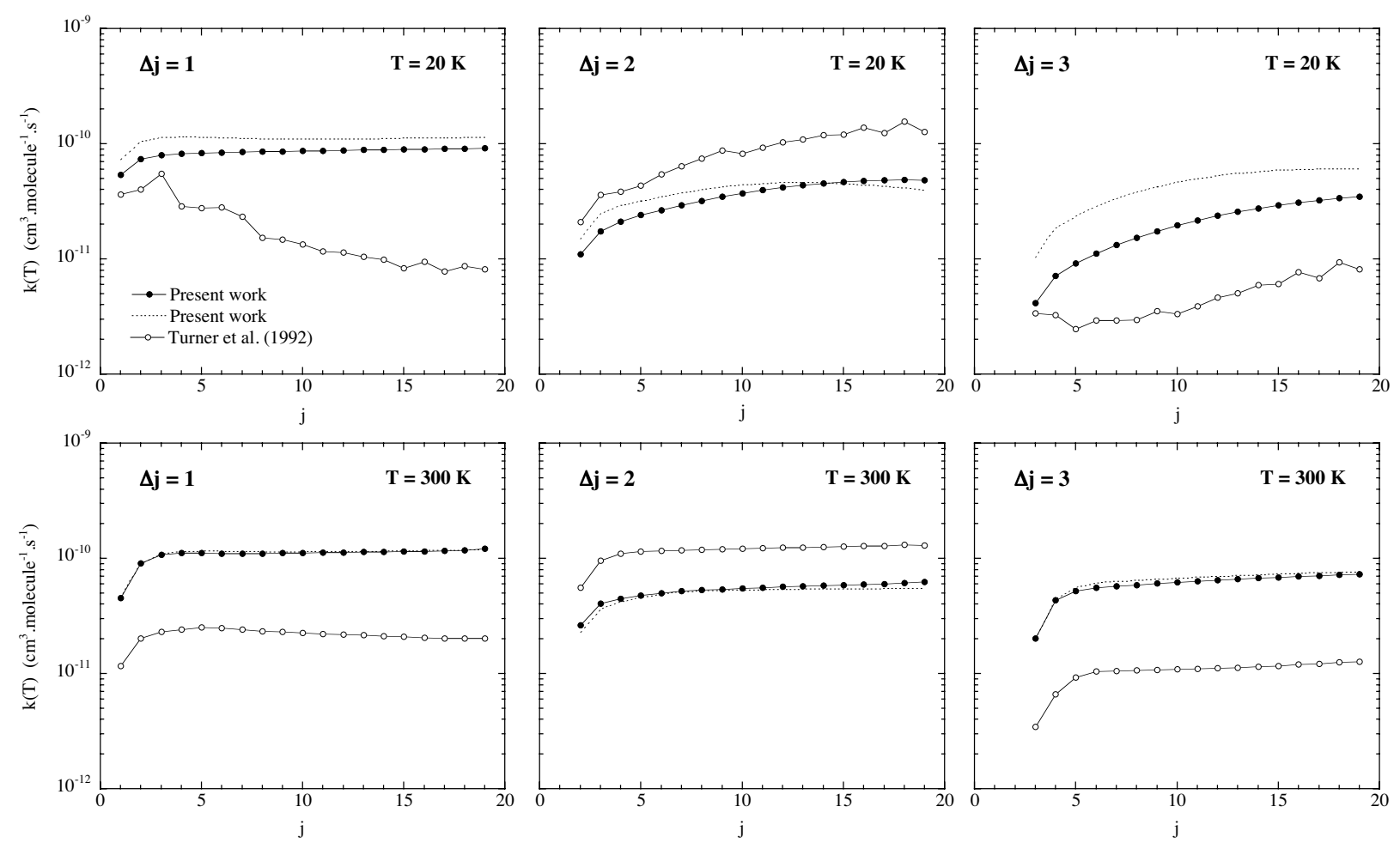

Fig. 7. Estimated values of the rotational de-excitation rate coefficients of $\mathrm{SiO}(j)$ in collision with para- $\mathrm{H}_{2}(j=0)$ obtained from: CC scattering calculations using the $\mathrm{SiO}-\mathrm{H}_{2}$ reduced mass and the $\mathrm{SiO}-\mathrm{He}$ ab initio PES (filled circles); $\mathrm{SiO}-\mathrm{He}$ de-excitation rate coefficients corrected from the square root of the reduced mass ratio $\mu_{\mathrm{SiO}-\mathrm{He}} / \mu_{\mathrm{SiO}-\mathrm{H}_{2}}$ (dotted line); coupled-states scattering calculations of Turner et al. (1992) using the SiO-H reduced mass and the SiO-He PES of Bieniek \& Green (1981) (empty circles).

A new 2D SiO-He PES was employed, computed by means of highly correlated ab initio calculations and extended basis sets. The overall features of the ab initio PES agree with the results of a new empirical model (Bartolomei et al. 2006), but differ significantly from those associated with the electron-gas PES of Bieniek \& Green (1981). Particularly, the asymmetric character of the $\mathrm{SiO}-\mathrm{He} \mathrm{PES}$ is found to be much more pronounced in the ab initio case, leading to large odd anisotropic terms in the polynomial expansion of the PES. Consequently, the inelastic cross sections resulting from both PESs show opposite propensity rules for even and odd $\Delta j$ transitions and differ significantly in their magnitudes. In the present case, the inelastic cross sections as well as the leading rate coefficients are found to be larger for odd $\Delta j$ transitions than for even ones.

Additional calculations were performed using the $\mathrm{SiO}-\mathrm{He}$ PES to compute approximated values of SiO-para- $\mathrm{H}_{2}(j=0)$ collisional rate coefficients for rotational levels up to $j=19$ and kinetic temperatures up to $300 \mathrm{~K}$. These rate coefficients have been compared first to the $\mathrm{SiO}-\mathrm{He}$ rate coefficients scaled by a factor of 1.4 to account for the different reduced mass of the two colliding systems. Although the two sets of rate coefficients agree within $10 \%$ for low $\Delta j$ transitions at high temperatures, differences up to a factor of 2 are found for higher $\Delta j$ transitions, that rise up to an order of magnitude for both high $\Delta j$ and low temperatures. Such a comparison allows us to assess the effect of the reduced mass on collision dynamics. Our calculated $\mathrm{SiO}$-para- $\mathrm{H}_{2}(j=0)$ collisional rate coefficients have been also compared to those of Turner et al. (1992) for which the SiO-He PES of Bieniek \& Green (1981) was employed. In such a case quite important discrepancies are found even for low $\Delta j$ transitions and high temperatures. For odd $\Delta j$ transitions our rate coefficients are larger by a factor of 5 , while for even $\Delta j$ transitions they are found to be smaller by about a factor of 2.5 . For higher $\Delta j$ transitions or low temperatures the discrepancies can rise up to an order of magnitude. Since both sets of rate coefficients rest upon identical approximations, this illustrates the need for a proper description of the PES to achieve accurate values of collisional rate coefficients.

The quite large discrepancies found between our results and those of Turner et al. (1992) for the SiO-para- $\mathrm{H}_{2}(j=0)$ colliding system may have an important impact on the diagnostic of column densities derived from $\mathrm{SiO}$ line modelling. Hence, given the improved accuracy of the present SiO-He PES, our collisional rate coefficient values are thought to be the most appropriate for an astrophysical purpose, until the $\mathrm{SiO}-\mathrm{H}_{2}$ colliding system is explicitly considered. Indeed, results on systems such as $\mathrm{CO}$ (Wernli et al. 2006) and $\mathrm{H}_{2} \mathrm{O}$ (Phillips et al. 1996), for which both collision partners were considered, showed that the value of 1.4 assumed for the scaling factor of rates with He was in fact within a range $1-3$. The sets of rotational (de)excitation rate coefficients for both the $\mathrm{SiO}-\mathrm{He}$ and $\mathrm{SiO}-$ para- $\mathrm{H}_{2}(j=0)$ colliding systems are available from the BASECOL web site ${ }^{3}$. The range of higher temperatures $(300-2000 \mathrm{~K})$ is currently under investigation to provide the ro-vibrational rate coefficients needed, for instance, in the case of circumstellar $\mathrm{SiO}$ maser line emissions from evolved stars.

Acknowledgements. The PES calculations were performed on the MPOPM cluster at the Paris-Meudon Observatory. Quantum scattering calculations were carried out at the IDRIS French national computing centre under project no. 060883. This work was supported by the EU FP6 Program "Molecular Universe". We would like to acknowledge F. Lique, F. Daniel, and A. Spielfiedel for the very helpful discussions, as well as M. Bartolomei for his contribution to this work. We also acknowledge G. Dhont for providing us the fitting code employed in the present study.

3 amdpo.obspm.fr/basecol/ 


\section{References}

Acord, J. M., Walmsley, C. M., \& Churchwell, E. 1997, ApJ, 475, 693

Arthurs, A. M., \& Dalgarno, A. 1963, Proc. Roy. Soc. A, 256, 540

Bartolomei, M., Cappelletti, D., \& Pirani, F. 2006, Private Communication

Bieging, J. H., Shaked, S., \& Gensheimer, P. D. 2000, ApJ, 543, 897

Bieniek, R. J., \& Green, S. 1981, Chem. Phys. Lett., 84, 380

Boys, S. F., \& Bernardi, F. 1970, Mol. Phys., 19, 553

Carty, D., Goddard, A., Sims, I. R., \& Smith, I. W. M. 2004, J. Chem. Phys., 121,4671

Cecchi-Pestellini, C., Bodo, E., Balakrishnan, N., \& Dalgarno, A. 2002, ApJ, 571, 1015

Chen, X., Shen, Z.-Q., Imai, H., \& Kamohara, R. 2006, ApJ, 640, 982

Christoffel, K. M., \& Bowman, J. M. 1983, J. Chem. Phys., 78, 3952

Codella, C., Bachiller, R., \& Reipurth, B. 1999, A\&A, 343, 585

Cybulski, S. M., \& Toczylowski, R. R. 1999, J. Chem. Phys., 111, 10520

Dayou, F., \& Spielfiedel, A. 2003, J. Chem. Phys., 119, 4237

Dunning, T. H. 1989, J. Chem. Phys., 90, 1007

Flower, D. R., \& Pineau des Forets, G. 1995, MNRAS, 275, 1049

Gibb, A. G., Richer, J. S., Chandler, C. J., \& Davis, C. J. 2004, ApJ, 603, 198

González Delgado, D., Olofsson, H., Kerschbaum, F., et al. 2003, A\&A, 411, 123

Gordon, R. G., \& Kim, Y. S. 1972, J. Chem. Phys., 56, 3122

Gueth, F., Guilloteau, S., \& Bachiller, R. 1998, A\&A, 333, 287

Herpin, F., Baudry, A., Thum, C., Morris, D., \& Wiesemeyer, H. 2006, A\&A, 450, 667

Honerjäger, R., \& Tischer, R. 1974, Z. Nature A, 29, 1695

Huber, K. P., \& Herzberg, G. 1979, Constants of Diatomic Molecules (New York: Van Nostrand Reinhold Co.)

Hutson, J. M., \& Green, S. 1994, MOLSCAT computer code version 14, distributed by Collaborative Computational Project No. 6 of the Engineering and Physical Sciences Research Council

Jeong, K. S., Winters, J. M., \& Sedlmayr, E. 1999, in IAU Symp., 191, Asymptotic Giant Branch Stars, ed. T. Le Bertre, A. Lebre, \& C. Waelkens, 233

Jiménez-Serra, I., Martín-Pintado, J., Rodríguez-Franco, A., \& Martín, S. 2005, ApJ, 627, L121

Kendall, R. A., Dunning, T. H., \& Harrison, R. J. 1992, J. Chem. Phys., 96, 6796

Knowles, P. J., \& Werner, H.-J. 1985, Chem. Phys. Lett., 115, 259

Le Picard, S. D., Canosa, A., Pineau des Forêts, G., Rebrion-Rowe, C., \& Rowe, B. R. 2001, A\&A, 372, 1064

Lique, F., Spielfiedel, A., \& Cernicharo, C. 2006, A\&A, 451, 1125
Lovas, F., Maki, A., \& Olson, W. 1981, J. Mol. Spectrosc., 87, 449

Manolopoulos, D. E. 1986, J. Chem. Phys., 85, 6425

Martin-Pintado, J., Bachiller, R., \& Fuente, A. 1992, A\&A, 254, 315

Martin-Pintado, J., de Vicente, P., Fuente, A., \& Planesas, P. 1997, ApJ, 482, L45

McCurdy, C. W., \& Miller, W. H. 1977, J. Chem. Phys., 67, 463

Nisini, B., Codella, C., Giannini, T., \& Richer, J. S. 2002, A\&A, 395, L25

Olney, T. N., Cann, N. M., \& Cooper, G. E. B. C. 1997, Chem. Phys., 223, 59

Pardo, J. R., Cernicharo, J., Gonzalez-Alfonso, E., \& Bujarrabal, V. 1998, A\&A, 329, 219

Phillips, T. R., Maluendes, S., \& Green, S. 1996, ApJS, 107, 467

Pirani, F., Cappelletti, D., \& Liuti, G. 2001, Chem. Phys. Lett., 350, 286

Ramonda, J. W., Muenter, J. S., \& Klemperer, W. A. 1970, J. Chem. Phys., 52, 3458

Reinsch, E.-A., \& Meyer, W. 1976, Phys. Rev. A, 14, 915

Schilke, P., Walmsley, C. M., Pineau des Forets, G., \& Flower, D. R. 1997, A\&A, 321,293

Schöier, F. L., Olofsson, H., Wong, T., Lindqvist, M., \& Kerschbaum, F. 2004, A\&A, 422, 651

Schöier, F. L., van der Tak, F. F. S., van Dishoeck, E. F., \& Black, J. H. 2005, A\&A, 432, 369

Sisak, M., \& Secrest, D. 1991, J. Chem. Phys., 94, 5488

Sisak, M., \& Secrest, D. 1992, J. Chem. Phys., 96, 230

Smith, L. N., Malik, D. J., \& Secrest, D. 1979, J. Chem. Phys., 71, 4502

Thomas, L., Kraemer, W., \& Diercksen, G. 1980, Chem. Phys., 51, 131

Turner, B. E., Chan, K.-W., Green, S., \& Lubowich, D. A. 1992, ApJ, 399, 114

Usero, A., García-Burillo, S., Martín-Pintado, J., Fuente, A., \& Neri, R. 2006, A\&A, 448, 457

Watts, J. D., Gauss, J., \& Bartlett, R. J. 1993, J. Chem. Phys., 98, 8718

Werner, H.-J., \& Meyer, W. 1976, Phys. Rev. A, 13, 13

Werner, H.-J., \& Knowles, P. J. 1985, J. Chem. Phys., 82, 5053

Werner, H.-J., Follmeg, B., Alexander, M. H., \& Lemoine, D. 1989, J. Chem. Phys., 91, 5425

Werner, H.-J., Knowles, P. J., Lindh, R., et al. 2003, MOLPRO, version 2002.6, a package of ab initio programs, see http: //www.molpro.net

Wernli, M., Valiron, P., Faure, A., et al. 2006, A\&A, 446, 367

Wilson, R. W., Jefferts, K. B., \& Penzias, A. A. 1970, ApJ, 161, L43

Wilson, R. W., Penzias, A. A., Jefferts, K. B., Kutner, M., \& Thaddeus, P. 1971, ApJ, 167, L97

Woon, D. E., \& Dunning, T. H. 1993, J. Chem. Phys., 98, 1358

Woon, D. E., \& Dunning, T. H. 1994, J. Chem. Phys., 100, 2975

Ziurys, L. M., Friberg, P., \& Irvine, W. M. 1989, ApJ, 343, 201 